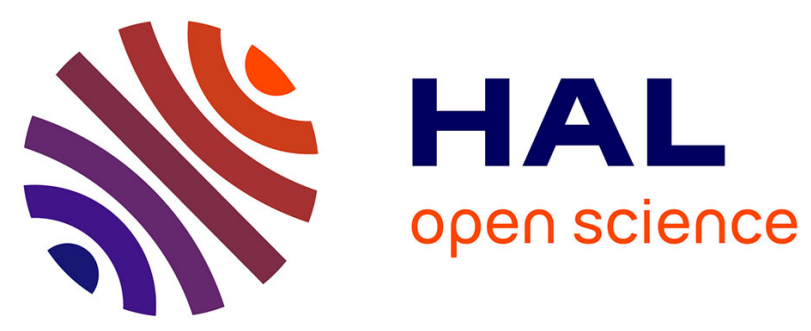

\title{
Observer design for a class of parabolic systems with arbitrarily delayed measurements
}

Tarek Ahmed-Ali, Emilia Fridman, Fouad Giri, Mohamed Kahelras, Françoise

Lamnabhi-Lagarrigue, Laurent Burlion

\section{- To cite this version:}

Tarek Ahmed-Ali, Emilia Fridman, Fouad Giri, Mohamed Kahelras, Françoise Lamnabhi-Lagarrigue, et al.. Observer design for a class of parabolic systems with arbitrarily delayed measurements. 2018 IEEE Conference on Decision and Control (CDC 2018), Dec 2018, Miami Beach, Fl, United States. pp.2199-2204, 10.1109/CDC.2018.8618938 . hal-02338989

\section{HAL Id: hal-02338989 \\ https://hal.science/hal-02338989}

Submitted on 31 May 2021

HAL is a multi-disciplinary open access archive for the deposit and dissemination of scientific research documents, whether they are published or not. The documents may come from teaching and research institutions in France or abroad, or from public or private research centers.
L'archive ouverte pluridisciplinaire HAL, est destinée au dépôt et à la diffusion de documents scientifiques de niveau recherche, publiés ou non, émanant des établissements d'enseignement et de recherche français ou étrangers, des laboratoires publics ou privés. 


\title{
Observer design for a class of parabolic systems with arbitrarily delayed measurements.
}

\author{
Tarek Ahmed-Ali ${ }^{1}$, Emilia Fridman ${ }^{2}$, Fouad Giri ${ }^{1}$, Mohamed Kahelras ${ }^{3}$, Francoise Lamnabhi-Lagarrigue ${ }^{3}$ \\ and Laurent Burlion ${ }^{4}$
}

\begin{abstract}
An observer is designed for a class of nonlinear parabolic PDEs with delayed point measurements. The novelty lies is that the delay size is arbitrary. To compensate for this arbitrary delay effect, the observer consists of several chained sub-observers. Each sub-observer compensates a fraction of the global delay. The resulting estimation error system is shown to be exponentially stable provided that a sufficient number of sub-observers is used. The stability analysis is based on a specific Lyapunov-Krasovskii functional and the stability conditions are expressed in terms of LMIs.
\end{abstract}

\section{INTRODUCTION}

The problem of designing observers for nonlinear systems containing delays has been a hot research topic during the past recent years. Existing works have been focused on finite-dimensional systems described by ODEs, see e.g. cite [1] and reference list therein. The dominant approach consisted in starting with an exponentially convergent state observer for the delay-free system and then modifying the observer so that exponential convergence is preserved in the presence of time-delay. The modification essentially consists in introducing output or state predictors to compensate for time delay. This has been illustrated with observers based on drift-observability property [2] or on high-gain observers [3], [1] , [4] . However, the involved predictors have proved to be useful in compensating the delay effect only up to some upper limit. To enlarge the maximum time-delay, a set of chained predictors simultaneously operating have to be implemented [2].

In parallel with the above "finite-dimensional" research activity, the "infinite-dimensional" backstepping transformation based approach has first been developed for linear systems, see e.g. [5] and references therein. This approach consists in letting the output sensor delay be captured by a first-order hyperbolic PDE. Then, full-order observers are designed that estimate both the system (finite-dimensional) state and the sensor

\footnotetext{
*This work was not supported by any organization

${ }^{1}$ Tarek Ahmed-Ali and Fouad Giri are with Laboratoire d'Automatique de Caen, Universit de Caen Normandie, ENSICAEN,14000 Caen, France, ahmed_alifr@yahoo.fr, fouadgiri@yahoo.fr

${ }^{2}$ Emilia Fridman with the School of Electrical Engineering, Tel-Aviv University, Tel-Aviv 69978, Israel emiliadeng.tau.ac.il

${ }^{3}$ Mohamed Kahelras and Francoise Lamnabhi-Lagarrigue are with Laboratoire des Signaux et Systmes (L2S), CNRS-CentraleSuplec-Université Paris Sud, Université Paris Saclay, 91192 Gif-sur-Yvette, France, mohamed.kahelras@l2s.centralesupelec.fr,

Francoise.Lamnabhi-Lagarrigued12s.centralesupelec.fr

${ }^{4}$ Laurent Burlion , ONERA, 31000 Toulouse,

Francelaurent.Burlion@onera.fr
}

(infinite-dimensional) state. The extension of this approach to (triangular) nonlinear systems has been studied in [6], where a high-gain type observer has been developed. The arbitrary-size delay effect has been compensated for by developing a PDE version of the chain observer concept. The problem of observer design for nonlinear PDEs with arbitrary delays measurements has yet to be solved. In this paper, the problem is addressed for a class of parabolic PDEs under point measurements as in [7]. In the latter paper the results were confined to small delays. To compensate for the effect of the arbitrary-size delay, the concept of chain-observer is extended to fit this class of systems. Accordingly, the initial delay PDE system representation is re-expressed in the form of fictive delayed subsystems. The observer is composed of elementary observers connected in series. The interconnection is such that the first elementary observer is directly driven by the physical system output. Then, the elementary observer is driven by a virtual output generated by the previous observer. Each elementary observer can be viewed as a predictor which compensates for the effects of the fractional time-delay. As in [7], using an appropriate Lyapunov-Krasovskii functional, sufficient conditions are established in terms of LMIs for the chain observer to be exponentially convergent. The sufficient conditions involve the minimal number of elementary observers: the larger the delay the larger the number of observers. Extension to sampled data delayed measurements is presented. The paper is organized as follows: first, the observation problem under study is formulated in Section 2 ; then, the observer design and analysis are dealt within Sections 3; a conclusion and reference list end the paper.

\section{Notations and preliminaries}

Throughout the paper the superscript $T$ stands for matrix transposition, $\mathbf{R}^{n}$ denotes the $\mathrm{n}$-dimensional Euclidean space with vector norm |.|, $\mathbf{R}^{n \times m}$ is the set of all $n \times m$ real matrices, and the notation $P>0$, for $P \in \mathbf{R}^{n \times n}$, means that $P$ is symmetric and positive definite. In matrices, symmetric terms are denoted $* ; \lambda_{\min }(P)\left(\operatorname{resp.} \lambda_{\max }(P)\right)$ denotes the smallest (resp. largest) eigenvalue. The notation $\left(t_{i}\right)_{i \geq 0}$ refers to a strictly increasing sequence such that $t_{0}=0$ and $\lim _{k \rightarrow \infty} t_{i}=\infty$. The sampling periods are bounded i.e. $0<t_{i+1}^{k \rightarrow \infty}-t_{i}<h$ for some scalar $0<h<\infty$ and all $i=0,1, \ldots, \infty$. We also define the variable $\tau(t)=t-t_{i}, t \in\left[t_{i}, t_{i+1}\right) . L_{2}(0, l)$ is the Hilbert space of square integrable functions $z(x), x \in[0, l]$ with the 
corresponding norm $\|z(x)\|_{L_{2}}=\sqrt{\int_{0}^{l} z^{2}(x) d x}$. $\mathcal{H}^{1}(0, l)$ is the Sobolev space of absolutely continuous functions $z:(0, l) \rightarrow \mathbf{R}$ with the square integrable derivative $\frac{d}{d x}$. $\mathcal{H}^{2}(0, l)$ is the Sobolev space of absolutely continuous functions $\frac{d z}{d x}:(0, l) \rightarrow \mathbf{R}$ and with $\frac{d^{2} w}{d x^{2}} \in L_{2}(0, l)$. Given a two-argument function $u(x, t)$, its partial derivatives are denoted $u_{t}=\frac{\partial u}{\partial t}, u_{x x}=\frac{\partial^{2} u}{\partial x^{2}}$. Throughout the paper the following lemma will be used to prove exponential convergence of our observer.

Lemma 1: ( Halanay's type Inequalities [9])

Let $0<\delta_{1}<2 \delta$ and let $V:\left[t_{0}-h, \infty\right) \rightarrow[0, \infty)$ be an absolutely continuous function which satisfies

$$
\dot{V}(t) \leq-2 \delta V(t)+\delta_{1} \sup _{-h \leq s \leq 0} V(t+s)
$$

Then

$$
V(t) \leq e^{-2 \alpha\left(t-t_{0}\right)} \sup _{-h \leq s \leq 0} V(t+s)
$$

where $\alpha$ is the unique positive solution of the equation

$$
\alpha=\delta-\frac{\delta_{1} e^{2 \alpha h}}{2}
$$

\section{SYSTEM DESCRIPTION}

We consider a semi-linear diffusion equation:

$$
u_{t}(x, t)=u_{x x}(x, t)+f(u(x, t), x, t)
$$

with Dirichlet conditions $u(0,0)=u(l, 0)=0$. The system output is, $y(t)=u\left(\bar{x}_{j}, t-D\right)$ where $\bar{x}_{j}=\frac{x_{j+1}+x_{j}}{2}(j=$ $0, \ldots, N-1)$ and the points $x_{j}$ divide the interval $[0, l]$ such that $0=x_{0}<\ldots<x_{N}=l$. It is supposed that $x_{j+1}-$ $x_{j} \leq \Delta$. The constant $D$ represents an arbitrarily delay and $N$ is the number of distributed sensors. It is also supposed that the function $f$ is known, of class $\mathcal{C}^{1}$, and satisfying $m_{f} \leq f_{u} \leq M_{f}$, for some scalar constants $m_{f}$ and $M_{f}$.

\section{OBSERVER DESIGN}

We will present an observer, which ensures exponential convergence for an arbitrarily delay $D$. This chain is constituted by $m$ sub-observers in cascade. Each sub-observer will estimate the state $u\left(x, t+\frac{k}{m} D-D\right)$ by using the estimation provided by the previous one in the chain. The last sub-observer in the chain provides the estimation of the $u(x, t)$. As we will see below, by using a suitable Lyapunov functional, we will derive sufficient conditions involving both delay $D$, and the number of sub-observers in the chain $m$.

As in [2] we introduce the following notations for the delayed states :

$$
\begin{gathered}
u^{0}(x, t)=u(x, t-D), \\
u^{k}(x, t)=u\left(x, t+\frac{k}{m} D-D\right), \quad k=1 \ldots, m
\end{gathered}
$$

Using these notations we easily check that :

$$
u^{k+1}(x, t)=u^{k}\left(x, t-\frac{D}{m}\right)
$$

and

$$
u^{m}(x, t)=u(x, t)
$$

where $m$ is the number of sub-observers in the considered chain.

We propose the following observer structure :

for $k=1$ :

$$
\begin{aligned}
\hat{u}_{t}^{1}(x, t)= & \hat{u}_{x x}^{1}(x, t)+f\left(\hat{u}^{1}(x, t), x, t\right) \\
- & L\left(\hat{u}^{1}\left(\bar{x}_{j}, t-\frac{D}{m}\right)-y(t)\right), \\
& \forall x \in\left[x_{j}, x_{j+1}\right)
\end{aligned}
$$

for $k=2, \ldots, m$ :

$$
\begin{aligned}
\hat{u}_{t}^{k}(x, t)= & \hat{u}_{x x}^{k}(x, t)+f\left(\hat{u}^{k}(x, t), x, t\right) \\
- & L\left(\hat{u}^{k}\left(\bar{x}_{j}, t-\frac{D}{m}\right)-\hat{u}^{k-1}\left(\bar{x}_{j}, t\right)\right), \\
& \forall x \in\left[x_{j}, x_{j+1}\right),
\end{aligned}
$$

It is readily checked that the observation error systems $e^{k}(x, t)=\hat{u}^{k}(x, t)-u^{k}(x, t)$ undergoes the following equations:

for $k=1$ :

$$
\begin{aligned}
e_{t}^{1}(x, t)= & e_{x x}^{1}(x, t)+f\left(\hat{u}^{1}(x, t), x, t\right)-f\left(u^{1}(x, t), x, t\right) \\
- & L e^{1}\left(\bar{x}_{j}, t-\frac{D}{m}\right), \\
& \forall x \in\left[x_{j}, x_{j+1}\right),
\end{aligned}
$$

for $k=2, \ldots, m$ :

$$
\begin{aligned}
e_{t}^{k}(x, t)= & e_{x x}^{k}(x, t)+f\left(\hat{u}^{k}(x, t), x, t\right)-f\left(u^{k}(x, t), x, t\right) \\
- & L\left(\hat{u}^{k}\left(\bar{x}_{j}, t-\frac{D}{m}\right)-\hat{u}^{k-1}\left(\bar{x}_{j}, t\right)\right), \\
& \forall x \in\left[x_{j}, x_{j+1}\right),
\end{aligned}
$$

Using the fact that

$$
u^{k}\left(x, t-\frac{D}{m}\right)=u^{k-1}(x, t)
$$

then, for $k=1$ :

$$
\begin{aligned}
e_{t}^{1}(x, t)= & e_{x x}^{1}(x, t)+f\left(\hat{u}^{1}(x, t), x, t\right)-f\left(u^{1}(x, t), x, t\right) \\
- & L e^{1}\left(\bar{x}_{j}, t-\frac{D}{m}\right) \\
& \forall x \in\left[x_{j}, x_{j+1}\right) \\
e^{1}(l, t)= & e^{1}(0, t)=0
\end{aligned}
$$

for $k=2, \ldots, m$ :

$$
\begin{aligned}
e_{t}^{k}(x, t)= & e_{x x}^{k}(x, t)+f\left(\hat{u}^{k}(x, t), x, t\right)-f\left(u^{k}(x, t), x, t\right) \\
- & L e^{k}\left(\bar{x}_{j}, t-\frac{D}{m}\right)+L e^{k-1}\left(\bar{x}_{j}, t\right), \\
& \forall x \in\left[x_{j}, x_{j+1}\right), \\
e^{k}(l, t)= & e^{k}(0, t)=0,
\end{aligned}
$$

which leads to : 
for $k=1$ :

$$
\begin{aligned}
e_{t}^{1}(x, t)= & e_{x x}^{1}(x, t)+\Psi\left(x, t, e^{1}\right)\left(\hat{u}^{1}(x, t)-u^{1}(x, t)\right) \\
- & L e^{1}\left(\bar{x}_{j}, t-\frac{D}{m}\right) \\
& x \in\left[x_{j}, x_{j+1}\right) \\
e^{1}(l, t)= & e^{1}(0, t)=0
\end{aligned}
$$

for $k=2, \ldots, m$ :

$$
\begin{aligned}
e_{t}^{k}(x, t)= & e_{x x}^{k}(x, t)+\Psi\left(x, t, e^{k}\right)\left(\hat{u}^{k}(x, t)-u^{k}(x, t)\right) \\
- & L e^{k}\left(\bar{x}_{j}, t-\frac{D}{m}\right)+L e^{k-1}\left(\bar{x}_{j}, t\right), \\
& \forall x \in\left[x_{j}, x_{j+1}\right), \\
e^{k}(l, t)= & e^{k}(0, t)=0,
\end{aligned}
$$

where

$$
\Psi\left(x, t, e^{k}\right)=\int_{0}^{1} f_{u}\left(\hat{u}^{k}+\theta e^{k}, x, t\right) d \theta
$$

Remark: The well-posedness for the system (3) and the error system (10)-(11) can be proven with the same arguments than those used in [7], see also [10]. For instance, consider

$$
w(t)=e^{1}(., t)
$$

of the error system (10). The equation (10) can be rewritten as a differential equation in the Hilbert space $H=L_{2}(0, l)$ with the norm $\|\cdot\|_{L_{2}}$

$$
\dot{w}(t)=A w(t)+F(t, w(t)), \quad t \geq 0
$$

where the operator $A$ is defined by:

$$
A=\frac{\partial^{2}}{\partial x^{2}}
$$

and has the dense domain:

$$
\mathcal{D}_{2}(A)=\left\{w \in \mathcal{H}^{2}(0, l): w(0)=w(l)=0\right\} .
$$

The nonlinear term $F: R \times \mathcal{H}^{2}(0, l) \rightarrow L_{2}(0, l)$ is defined on functions $w(., t)$ as:

$$
\begin{aligned}
F(t, w(., t))= & \Psi(x, t, w(x, t)) w(x, t)-L w\left(\bar{x}_{j}, t-\frac{D}{m}\right) \\
= & \Psi(x, t, w(x, t)) w(x, t)-L w\left(x, t-\frac{D}{m}\right) \\
& +L \int_{\bar{x}_{j}}^{x} w_{\xi}\left(\xi, t-\frac{D}{m}\right) d \xi d x
\end{aligned}
$$

Let us also define the following Hilbert space

$$
\mathcal{D}_{1}(A)=\left\{w \in \mathcal{H}^{1}(0, l): w(0)=w(l)=0\right\},
$$

Using relevant material on fractional operators degrees [11] and Henry's theorem [12] , we get: if $w\left(t_{0}\right) \in \mathcal{D}_{1}(A)$, then

$$
w(t)-w\left(t_{0}\right)=\int_{t_{0}}^{t}[A w(s)+F(s, w(s))] d s
$$

holds for all $t \geq t_{0}$.

Theorem 1: Given $D$ and $m$, consider the system (3) and the observer (4)-(5). Given positive constants $\Delta, \delta, L>$
$M_{f}-\frac{\pi^{2}}{l^{2}}, R$ and $\delta_{1}$ such that $2 \delta>\delta_{1}$, let there exist positive scalars $p_{1}, p_{2}, p_{3}, r$ and $g$ such that :

$$
\delta p_{3}<p_{2} ; \frac{\Delta}{\pi} L R^{-1}\left(p_{3}+p_{2}\right)<\delta_{1} p_{3}
$$

and

$$
\Phi_{m_{f}}<0 ; \Phi_{M_{f}}<0
$$

where

$$
\Phi_{\phi}=\left(\begin{array}{ccc}
\Phi_{11}-\lambda & \Phi_{12} & \Phi_{13} \\
\Phi_{12} & \Phi_{22} & \Phi_{23} \\
\Phi_{13} & \Phi_{23} & \Phi_{33}
\end{array}\right)
$$

with

$$
\begin{aligned}
\Phi_{11} & =2 \delta p_{1}+g-r e^{-2 \delta \frac{D}{m}}+2 p_{2}\left(\phi+\frac{\Delta}{2 \pi} L R\right) \\
\Phi_{12} & =-p_{2}+p_{1}+p_{3} \phi \\
\Phi_{13} & =r e^{-2 \delta \frac{D}{m}}-p_{2} L \\
\Phi_{22} & =\frac{\Delta L R p_{3}}{\pi}-2 p_{3}+r\left(\frac{D}{m}\right)^{2} \\
\Phi_{23} & =-L p_{3} \\
\Phi_{33} & =-(r+g) e^{-2 \delta \frac{D}{m}} \\
\lambda & =\frac{2 \pi^{2}}{l^{2}}\left(p_{2}-\delta p_{3}\right) .
\end{aligned}
$$

Then all the observation errors $\int_{0}^{1}\left(e^{k}(x, t)\right)^{2} d x$ and $\int_{0}^{1}\left(e_{x}^{k}(x, t)\right)^{2} d x \quad(k=1, . ., m)$ globally exponentially decay to zero as $t \rightarrow+\infty$. The above LMIs are always feasible for large enough $m$.

Proof :

The proof of the above theorem, will be performed by induction.

For $k=1$ consider the first observation error :

$$
\begin{aligned}
e_{t}^{1}(x, t)= & e_{x x}^{1}(x, t)+\Psi\left(x, t, e^{1}\right)\left(\hat{u}^{1}(x, t)-u^{1}(x, t)\right) \\
- & L e^{1}\left(\bar{x}_{j}, t-\frac{D}{m}\right), \\
& \forall x \in\left[x_{j}, x_{j+1}\right), \\
e^{1}(l, t)= & e^{1}(0, t)=0
\end{aligned}
$$

and the following Lyapunov-Krasovskii functional as in [7]

$$
\begin{aligned}
V^{1}(t) & =p_{1} \int_{0}^{l}\left(e^{1}(x, t)\right)^{2} d x+p_{3} \int_{0}^{l}\left(e_{x}^{1}(x, t)\right)^{2} d x \\
& +g \int_{0}^{l}\left[\int_{t-\frac{D}{m}}^{t} e^{2 \delta(s-t)}\left(e^{1}(x, s)\right)^{2} d s\right] d x \\
& +\frac{D}{m} r \int_{0}^{l}\left[\int_{-\frac{D}{m}}^{0} \int_{t+\theta}^{t} e^{2 \delta(s-t)}\left(e_{s}^{1}(x, s)\right)^{2} d s d \theta\right] d x
\end{aligned}
$$


As in [7], differentiating the above functional we find:

$$
\begin{aligned}
\dot{V}^{1}(t)+2 \delta V^{1}(t) & =2 p_{1} \int_{0}^{l} e^{1}(x, t) e_{t}^{1}(x, t) d x \\
& +2 p_{3} \int_{0}^{l} e_{x}^{1}(x, t) e_{x t}^{1}(x, t) d x \\
& -\frac{D}{m} r \int_{0}^{l} \int_{t-\frac{D}{m}}^{t} e^{2 \delta(s-t)} e_{s}^{1}(x, s)^{2} d s d x \\
& +\int_{0}^{l}\left[( \frac { D } { m } ) ^ { 2 } r \left(e_{t}^{1}(x, t)^{2}+g\left(e^{1}(x, t)\right)^{2}\right.\right. \\
& \left.-g e^{-2 \delta \frac{D}{m}}\left(e^{1}\left(x, t-\frac{D}{m}\right)\right)^{2}\right] d x \\
& +2 \delta p_{1} \int_{0}^{l}\left(e^{1}(x, t)\right)^{2} d x \\
& +2 \delta p_{3} \int_{0}^{l}\left(e_{x}^{1}(x, t)\right)^{2} d x
\end{aligned}
$$

using the descriptor method [13] for each $j$ and by summing these expressions for all $j=0, . ., N-1$, this leads to:

$$
\begin{aligned}
0 & =2 \int_{0}^{l}\left[p_{2} e^{1}(x, t)+p_{3} e_{t}^{1}(x, t)\right]\left[-e_{t}^{1}(x, t)+e_{x x}^{1}(x, t)\right. \\
& \left.+\Psi\left(x, t, e^{1}\right) e^{1}(x, t)-L e^{1}\left(x, t-\frac{D}{m}\right)\right] d x \\
& +2 \sum_{j=0}^{N-1} \int_{x_{j}}^{x_{j+1}}\left[p_{2} e^{1}(x, t)+p_{3} e_{t}^{1}(x, t)\right] \\
& \times L \int_{\bar{x}_{j}}^{x} e_{\xi}^{1}\left(\xi, t-\frac{D}{m}\right) d \xi d x
\end{aligned}
$$

Using Wirtinger inequality:

$$
\begin{aligned}
& -\quad \frac{D}{m} r \int_{0}^{l} \int_{t-\frac{D}{m}}^{t} e^{2 \delta(s-t)} e_{s}^{1}(x, s)^{2} d s d x \leq \\
& -r \quad \int_{0}^{l} e^{-2 \delta \frac{D}{m}}\left(\int_{t-\frac{D}{m}}^{t} e^{2 \delta(s-t)} e_{s}^{1}(x, s) d s\right)^{2} d x
\end{aligned}
$$

Here $p_{2}$ and $p_{3}$ are free parameters. Combining (25)-(26), we deduce by using the classical inequalities (Jesnsen, Young and Wirtinger) that:

$$
\begin{aligned}
\dot{V}^{1}(t) & +2 \delta V^{1}(t) \leq \int_{0}^{l} \eta^{T} \Phi_{\phi} \eta d x \\
& +\frac{\Delta}{\pi} L R^{-1}\left(p_{3}+p_{2}\right) \int_{0}^{l}\left(e_{x}^{1}\left(x, t-\frac{D}{m}\right)\right)^{2} d x
\end{aligned}
$$

where $\eta=\operatorname{col}\left\{e^{1}(x, t), e_{t}^{1}(x, t), e^{1}\left(x, t-\frac{D}{m}\right)\right\}$. Since $\Phi_{\phi}$ is affine in $\Phi$, then under (20):

$$
\int_{0}^{l} \eta^{T} \Phi_{\phi} \eta d x \leq 0,
$$

From this we also deduce

$$
\begin{aligned}
\dot{V}^{1}(t) & +2 \delta V^{1}(t)-\delta_{1} V^{1}\left(t-\frac{D}{m}\right) \leq \int_{0}^{l} \eta^{T} \Phi_{\phi} \eta d x \\
& +\left(\frac{\Delta}{\pi} L R^{-1}\left(p_{3}+p_{2}\right)-\delta_{1}\right) \int_{0}^{l}\left(e_{x}^{1}\left(x, t-\frac{D}{m}\right)\right)^{2} d x
\end{aligned}
$$

Then we conclude under conditions of Theorem 1, that

$$
\dot{V}^{1}(t)+2 \delta V^{1}(t)-\delta_{1} V^{1}\left(t-\frac{D}{m}\right) \leq 0
$$

By Lemma $1, V^{1}(t)$ is exponentially vanishing to zero.

Now by induction, let us suppose that the exponential convergence is guaranteed for $k-1$, and consider the observation for $k$. For this case the observation error is described by the following equation :

$$
\begin{aligned}
e_{t}^{k}(x, t)= & e_{x x}^{k}(x, t)+\Psi\left(x, t, e^{k}\right) e^{k}(x, t) \\
- & L e^{k}\left(\bar{x}_{j}, t-\frac{D}{m}\right)+L \int_{0}^{\bar{x}_{j}} e_{x}^{k-1}(x, t) d x, \\
& \forall x \in\left[x_{j}, x_{j+1}\right), \\
e^{k}(l, t)= & e^{k}(0, t)=0,
\end{aligned}
$$

The only difference between the above system and the one of the case $k=1$ is in the disturbing term $\int_{0}^{\bar{x}_{j}} e_{x}^{k-1}(x, t) d x$ which is supposed by induction exponentially vanishing to zero. By using the following Lyapunov-Krasovskii functional

$$
\begin{aligned}
V^{k}(t) & =p_{1} \int_{0}^{l}\left(e^{k}(x, t)\right)^{2} d x+p_{3} \int_{0}^{l}\left(e_{x}^{k}(x, t)\right)^{2} d x \\
& +g \int_{0}^{l}\left[\int_{t-\frac{D}{m}}^{t} e^{2 \delta(s-t)}\left(e^{k}(x, s)\right)^{2} d s\right] d x \\
& +\frac{D}{m} r \int_{0}^{l}\left[\int_{-\frac{D}{m}}^{0} \int_{t+\theta}^{t} e^{2 \delta(s-t)}\left(e_{s}^{k}(x, s)\right)^{2} d s d \theta\right] d x
\end{aligned}
$$

and similar arguments as for the case $k=1$, one can easily deduce that both $\int_{0}^{l}\left(e^{k}(x, t)\right)^{2} d x$ and $\int_{0}^{l}\left(e_{x}^{k}(x, t)\right)^{2} d x$ converge exponentially to zero. For large delays $D$, the feasibility of the LMIs (19) and (20) is always preserved for large enough $m$ [10].

\section{A. Extension to sampled-data case}

In this part, we present briefly the extension of the above observer to sampled- measurements case. In this case the output is available only at sampling instants $t_{i}$

$$
0=t_{0}<t_{1}<\ldots<t_{i}<\ldots, \quad \lim _{k \rightarrow \infty} t_{i}=\infty .
$$

We assume that the sampling intervals may be variable, but upper-bounded by a known bound $h$ :

$$
t_{i+1}-t_{i} \leq h \quad \forall i=0,1, \ldots
$$

The proposed observer has the following form : for $k=1$ :

$$
\begin{aligned}
\hat{u}_{t}^{1}(x, t)= & \hat{u}_{x x}^{1}(x, t)+f\left(\hat{u}^{1}(x, t), x, t\right) \\
- & L\left(\hat{u}^{1}\left(\bar{x}_{j}, t_{i}-\frac{D}{m}\right)-y\left(t_{i}\right)\right), \\
& \forall t \in\left[t_{i}, t_{i+1}\right), \quad \forall x \in\left[x_{j}, x_{j+1}\right),
\end{aligned}
$$


for $k=2, \ldots, m$ :

$$
\begin{aligned}
\hat{u}_{t}^{k}(x, t)= & \hat{u}_{x x}^{k}(x, t)+f\left(\hat{u}^{k}(x, t), x, t\right) \\
- & L\left(\hat{u}^{k}\left(\bar{x}_{j}, t-\frac{D}{m}\right)-\hat{u}^{k-1}\left(\bar{x}_{j}, t\right)\right), \\
& \forall t \in\left[t_{i}, t_{i+1}\right), \quad \forall x \in\left[x_{j}, x_{j+1}\right),
\end{aligned}
$$

Then the observation error is described by the following equations :

for $k=1$ :

$$
\begin{aligned}
e_{t}^{1}(x, t)= & e_{x x}^{1}(x, t)+\Psi\left(x, t, e^{1}\right)\left(\hat{u}^{1}(x, t)-u^{1}(x, t)\right) \\
- & L e^{1}\left(\bar{x}_{j}, t_{i}-\frac{D}{m}\right), \\
& \forall t \in\left[t_{i}, t_{i+1}\right), \quad \forall x \in\left[x_{j}, x_{j+1}\right) \\
e^{1}(l, t)= & e^{1}(0, t)=0
\end{aligned}
$$

for $k=2, \ldots, m$ :

$$
\begin{aligned}
e_{t}^{k}(x, t)= & e_{x x}^{k}(x, t)+\Psi\left(x, t, e^{k}\right)\left(\hat{u}^{k}(x, t)-u^{k}(x, t)\right) \\
- & L e^{k}\left(\bar{x}_{j}, t-\frac{D}{m}\right)+L e^{k-1}\left(\bar{x}_{j}, t\right) \\
& \forall t \in\left[t_{i}, t_{i+1}\right), \quad \forall x \in\left[x_{j}, x_{j+1}\right) \\
e^{k}(l, t)= & e^{k}(0, t)=0,
\end{aligned}
$$

As we can easily see, the unique difference with the observer without sampling measurements is for the first sub-observer $(k=1)$. In order to study the convergence of the case $k=1$, we use the following modified Lyapunov-Krasvoskii inspired from [8], [14]:

$$
\begin{aligned}
V^{1}(t) & =p_{1} \int_{0}^{l}\left(e^{1}(x, t)\right)^{2} d x+p_{3} \int_{0}^{l}\left(e_{x}^{1}(x, t)\right)^{2} d x \\
& +g \int_{0}^{l}\left[\int_{t-\frac{D}{m}}^{t} e^{2 \delta(s-t)}\left(e^{1}(x, s)\right)^{2} d s\right] d x \\
& +\frac{D}{m} r \int_{0}^{l}\left[\int_{-\frac{D}{m}}^{0} \int_{t+\theta}^{t} e^{2 \delta(s-t)}\left(e_{s}^{1}(x, s)\right)^{2} d s d \theta\right] d x \\
& +W h^{2} e^{2 \delta h} \int_{0}^{l} \int_{t_{i}-\frac{D}{m}}^{t} e^{2 \delta(s-t)}\left(e_{s}^{1}(x, s)\right)^{2} d s d x \\
& -\frac{\pi^{2}}{4} W \int_{0}^{l} \int_{t_{i}-\frac{D}{m}}^{t-\frac{D}{m}} e^{2 \delta(s-t)}\left[e^{1}(x, s)\right. \\
& \left.-e^{1}\left(x, t_{i}-\frac{D}{m}\right)\right]^{2} d s d x
\end{aligned}
$$

By generalized Wirtinger's inequality [14], the W-terms expression in $V^{1}$,

$$
\begin{gathered}
h^{2} e^{2 \delta h} \int_{0}^{l} \int_{t_{i}-\frac{D}{m}}^{t} e^{2 \delta(s-t)}\left(e_{s}^{1}(x, s)\right)^{2} d s d x \\
-\frac{\pi^{2}}{4} \int_{0}^{l} \int_{t_{i}-\frac{D}{m}}^{t-\frac{D}{m}} e^{2 \delta(s-t)}\left[e^{1}(x, s)-e^{1}\left(x, t_{i}-\frac{D}{m}\right)\right]^{2} d s d x
\end{gathered}
$$

is nonnegative and does not grow in the jumps [14]. By differentiating it, we obtain:

$$
\begin{aligned}
& h^{2} e^{2 \delta h} \int_{0}^{l}\left(e_{t}^{1}(x, s)\right)^{2} d x \\
- & 2 \delta h^{2} e^{2 \delta h} \int_{0}^{l} \int_{t_{i}-\frac{D}{m}}^{t} e^{2 \delta(s-t)}\left(e_{s}^{1}(x, s)\right)^{2} d s d x \\
- & \frac{\pi^{2}}{4} \int_{0}^{l}\left[e^{1}\left(x, t-\frac{D}{m}\right)-e^{1}\left(x, t_{i}-\frac{D}{m}\right)\right]^{2} d x \\
+ & \frac{\pi^{2}}{2} \delta \int_{0}^{l} \int_{t_{i}-\frac{D}{m}}^{t-\frac{D}{m}} e^{2 \delta(s-t)}\left[e^{1}(x, s)-e^{1}\left(x, t_{i}-\frac{D}{m}\right)\right]^{2} d s d x
\end{aligned}
$$

Using the same computation as in [14], we can easily derive the following theorem:

Theorem 2: Given $D, h$ and $m$, consider the system (3) and the observer (34)-(35). Given positive constants scalars $\Delta, \delta, L>M_{f}-\frac{\pi^{2}}{l^{2}}, R$ and $\delta_{1}$ such that $2 \delta>\delta_{1}$, let there exist positive scalars $p_{1}, p_{2}, p_{3}, r, W$ and $g$ such that :

$$
\delta p_{3}<p_{2} ; \frac{\Delta}{\pi} L R^{-1}\left(p_{3}+p_{2}\right)<\delta_{1} p_{3}
$$

and

$$
\Phi_{m_{f}}<0 \quad \Phi_{M_{f}}<0
$$

where

$\Phi_{\phi}=\left(\begin{array}{cccc}\Phi_{11}-\lambda & \Phi_{12} & \Phi_{13} & p_{2} L \\ \Phi_{12} & \Phi_{22}+W h^{2} e^{2 \delta h} & \Phi_{23} & p_{3} L \\ \Phi_{13} & \Phi_{23} & \Phi_{33} & 0 \\ p_{2} L & p_{3} L & 0 & -W \frac{\pi^{2}}{4}\end{array}\right)$

with $\Phi_{11}, \Phi_{12}, \Phi_{13}, \Phi_{22}, \Phi_{23}$ and $\Phi_{33}$ given by (22).

Then all the observation errors $\int_{0}^{1}\left(e^{k}(x, t)\right)^{2} d x$ and $\int_{0}^{1}\left(e_{x}^{k}(x, t)\right)^{2} d x \quad(k=1, \ldots, m)$ globally exponentially decay to zero as $t \rightarrow+\infty$.

\section{EXAMPLE}

Let us consider the following example :

$$
u_{t}=u_{x x}(x, t)+1.02 \pi^{2} u(x, t)
$$

with $u(x, 0)=\sin (x)$ and let $y_{j}=u\left(\bar{x}_{j}, t-D\right), j=$ $1, \ldots, N-1$, where $D$ is an arbitrarily delay and $\hat{u}(x, 0)=0$.

\section{Continuous case}

We choose $L=1, \Delta=\frac{1}{50}, \delta=0.21$ and $\delta_{1}=0.1$. The following table shows the value of the delay $D$ and the corresponding number of sub-observers $m$ for which the LMIs of Theorem 1 are feasible:

\begin{tabular}{|l|lllll|}
\hline$D$ & 0.42 & 0.85 & 1.2 & 1.70 & 2.13 \\
\hline$m$ & 1 & 2 & 3 & 4 & 5 \\
\hline
\end{tabular}

Numerical simulations of the above example are presented in the following. These figures show the state $u(x, t)$ of the system (44) and its estimates at the points $x=0.1$ and $x=0.6$. These results show that the state of the designed observer $\hat{u}(x, t)$ converges to $u(x, t)$ when $D=1 s$ using only 1 sub-observer $m=1$. However, when $D=2 s$, 
2 sub-observers $m=2$ are required to get satisfactory results. Further simulations show that the observer (10)-(11) estimates the state of the system (44) for larger delays than those presented in the previous table. Future works will be dedicated to the improvement of the sufficient conditions presented here by the LMIs (19) and (20).
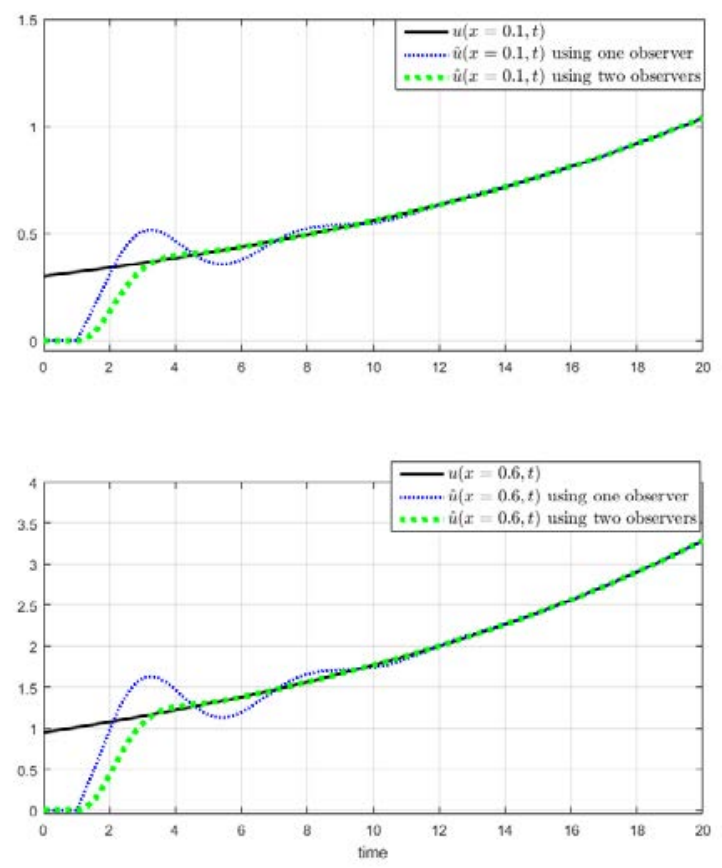

Fig. 1. The state $u(x, t)$ and its observations for $m=1$ and $m=2$ at $x=0.1$ and $x=0.6$ for a delav $D=1 s$
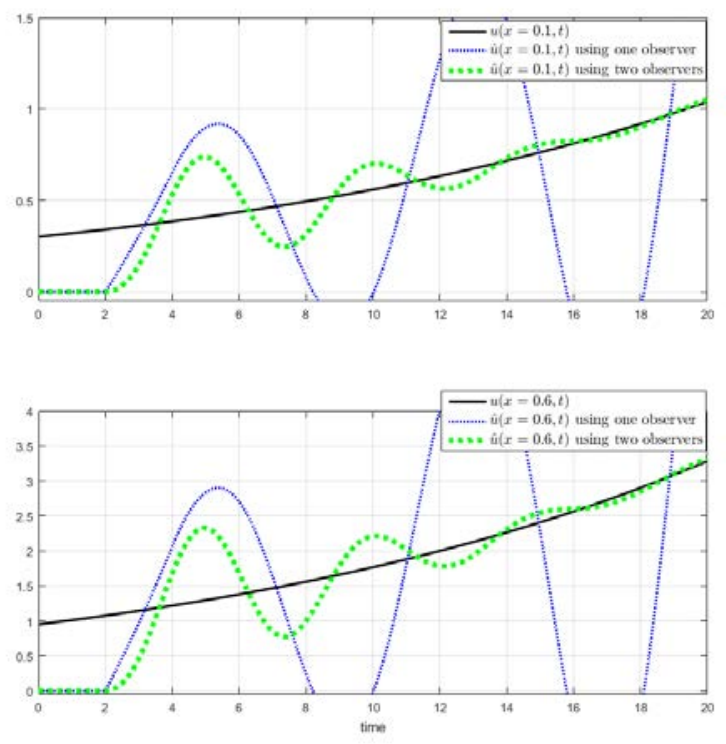

Fig. 2. The state $u(x, t)$ and its observations for $m=1$ and $m=2$ at $x=0.1$ and $x=0.6$ for a delay $D=2 s$

\section{Sampled Data Case}

We consider the case where the output $y_{j}, j=1, \ldots, N-1$, are periodically sampled with period $h$. We use the same values of the parameters as for the continuous case. The following tables show, for different values of the delay $D$, the value of the sampling period $h$ and the corresponding number of sub-observers $m$ for which the LMIs of Theorem 2 are feasible.

for $D=0.4$ :

\begin{tabular}{|c|llll|}
\hline$h$ & 0.05 & 0.37 & 0.47 & 0.51 \\
\hline$m$ & 1 & 2 & 3 & 4 \\
\hline
\end{tabular}

for $D=0.8$ :

\begin{tabular}{|c|llll|}
\hline$h$ & 0.05 & 0.1 & 0.37 & 0.5 \\
\hline$m$ & 2 & 3 & 4 & 8 \\
\hline
\end{tabular}

for $D=1.5$ :

\begin{tabular}{|c|lccc|}
\hline$h$ & 0.05 & 0.1 & 0.3 & 0.5 \\
\hline$m$ & 4 & 5 & 7 & 14 \\
\hline \multicolumn{5}{c}{ V. CONCLUSION }
\end{tabular}

In this paper, a novel observer is proposed for a class of parabolic systems. The main advantage of the proposed algorithm is that it can handle arbitrary delay and sampled measurements . This result can be easily extended to classes of cascade ODE/ parabolic PDE. The most disadvantage of the above algorithm is that it needs a chain of PDEs, which can lead to some implementation issues. The simplification of this observer is under investigation.

\section{REFERENCES}

[1] Cacace, F., Germani, A., and Manes, C. (2014). Nonlinear systems with multiple time-varying measurement delays. SIAM J. Control Optim., 52(3), 1862-1885.

[2] Germani, A., Manes, C., and Pepe, P. (2002). A new approach to state observation of nonlinear systems with delayed output. IEEE Transactions on Automatic Control, 47(1), 96-101.

[3] Ahmed-Ali, T., Cherrier, E., and Lamnabhi-Lagarrigue, F. (2012). Cascade high predictors for a class of nonlinear systems. IEEE Trans. on Aut. Control, 57, 224-229.

[4] Besançon, G., Georges, D., and Benayache, Z. (2007). Asymptotic state prediction for contiunous-time systems with delayed input and application to control. The Proceedings of the European Control Conference, 2-5.

[5] Krstic, M. (2009). Delay Compensation for Nonlinear, Adaptive, and PDE Systems. Birkhauser.

[6] Ahmed-Ali, T., Giri, F., Krstic, M., Kahelras, M. (2018). "PDE based observer design for nonlinear systems with large output delay," Systems \& Control Letters, 113, 1-8,

[7] Fridman, E. and Blighovsky, A. (2012). Robust sampled-data control of a class of semilinear parabolic systems. Automatica, 48, 826-836.

[8] Liu, K. and Fridman, E. (2012). Wirtinger inequality and lyapunov-based sampled-data stabilization. Automatica, 48, 102-108.

[9] Halanay,A. (1966). Differential Equations: Stability, Oscillations, Time Lags. Academic Press, New York.

[10] Ahmed-Ali, T., Fridman, E., Giri, F., Burlion, L. and Lamnabhi-Lagarrigue, F. (2016). Using exponential time-varying gains for sampled-data stabilization and estimation, Automatica, 67, 244-251.

[11] Tucsnak, M., \& Weiss, G. (2009). Observation and control for operator semigroups. Birkhauser.

[12] Henry, D. (1993). Geometric theory of semilinear parabolic equations. New York: Springer-Verlag.

[13] Fridman, E. (2014). Systems and Control: Foundations and Applications, Introduction to Time-Delay Systems: Analysis and Control. Birkhauser.

[14] Selivanov, A., Fridman, E. (2016). Observer-based input-to-state stabilization of networked control systems with large uncertain delays. Automatica , 74, 63-70. 